\title{
Notas sobre la acción exterior de las Regiones italianas en la jurisprudencia de la Corte Costituzionale
}

\author{
José Luis Blasco Díaz \\ Universitat Jaume I de Castellón
}

Sumario: I. INTRODUCCIÓN. II. LA ACCIÓN EXTERIOR DE LAS REGIONES Y PROVINCIAS AUTÓNOMAS. 1. Las actividades promocionales. 2. Las actividades de mero alcance internacional. III. EL REQUISITO NECESARIO DE LA COMUNICACIÓN PREVIA AL GOBIERNO DE LA REPÚBLICA

\section{INTRODUCCIÓN}

La República italiana ha conocido desde un tiempo a esta parte un importante incremento de la actividad internacional de sus Regiones y Provincias Autónomas, consecuencia tanto de su voluntad de realizar labores de promoción en el extranjero con el fin de reforzar su desarrollo, principalmente económico - aunque también cultural y social-, como de las transformaciones que viene experimentando la política europea en relación con las regiones que componen sus Estados miembros ${ }^{~}$. A partir de este fenómeno, común por lo demás en diferentes Estados europeos, la Corte Costituzionale italiana ha tenido que pronunciarse en abundantes ocasiones sobre diversas cuestiones incidentes en el desarrollo de tales actividades, fijando en su labor los límites de las mismas y resolviendo numerosos conflictos de atribuciones.

Hasta hace una década, se venía manteniendo por la jurisprudencia constitucional la existencia de una reserva estatal en materia de relaciones internacionales, comprensiva de todas ellas y considerada en términos de exclusividad, que conllevaba la facultad de determinar las directrices de la política exterior y de estipular pactos y acuerdos con otros sujetos de Derecho Internacional. En virtud de esta interpretación, se vetaba cualquier actividad en el extranjero que pretendieran llevar a término los entes locales italianos, por cuanto podía interferir en aquella atribución exclusiva. En este sentido, la participación externa ha estado vedada du-

I Por ejemplo, mediante la creación del Comité de las Regiones, regulado por los artículos 198A a 198C del Tratado de la Comunidad Europea, e incluido por el artículo G-E, 67, del Tratado de la Unión Europea. 
rante largo tiempo a las Regiones, e incluso las relaciones con la Comunidad Europea eran asimiladas a las propiamente internacionales y sujetas por ello a severos límites ${ }^{2}$.

No obstante, una oportunidad para que pudieran actuar en el exterior se abrió por medio del Decreto del Presidente de la República 616/1977, de 24 de julio ${ }^{3}$, que estableció en su artículo 4.2 la posibilidad de que las Regiones desarrollaran en el extranjero actividades promocionales relativas a las materias de su competencia, previo acuerdo con el Gobierno y en el ámbito de las directrices y de los actos de coordinación dictados por este último. De este modo, quedaba libre un ámbito en el que se permitía a las Regiones llevar a cabo diversas acciones de carácter internacional y de contenido heterogéneo, con el límite de que en su ejercicio no se pudiesen estipular acuerdos que derivasen en obligaciones jurídicas a cargo de los contrayentes, de las cuales los órganos centrales del Estado pudieran ser llamados a responder.

Al hilo de la brecha así abierta, años después la propia Corte Costituzionale, con su Sentencia 179/1987, de 22 de mayo, relativizó el anterior razonamiento, sustituyéndolo por una interpretación más restringida de aquello que debían ser las relaciones internacionales competencia del Estado. En ella, sin embargo, se reafirma en su precedente jurisprudencia, según la cual, en principio, es competencia de los órganos centrales del Estado el poder de determinar las directrices de política exterior en sentido extenso. Esta atribución viene inducida por el carácter unitario e indivisible de la República italiana, establecido en el artículo 5 de su Constitución, que condiciona y subordina las autonomías locales hasta el punto de despojarlas de cualquier potestad de decisión sobre la instauración o gestión de aquellas relaciones. Ello se preveía ya en el artículo 4 del citado DPR 616/1977, donde se dispone expresamente que el Estado debe ejercer las funciones, incluso en las materias transferidas o delegadas, relativas a las relaciones internacionales. Éstas son, por tanto, de competencia estatal en cuanto se refieran a la actividad política, económica, social o cultural de la República en relación con los otros Estados, y encuentran su origen en pactos estipulados y acuerdos suscritos por sujetos de Derecho Internacional, con la asunción de obligaciones de las cuales deben responder como tales.

\footnotetext{
2 Gómez Campo, La participación de las Comunidades Autónomas en los asuntos comunitarios europeos, Madrid, 1995, pág. 118.

3 Véase sobre la importancia de esta norma en la normalización de las relaciones entre las Regiones y el poder central a PINAR MAÑas, Las relaciones entre el Estado y las Regiones. La experiencia italiana, Madrid, 1986, págs. 57 y ss.
} 
Sin embargo, dice la Corte, el sistema constitucional italiano permite explícitamente que el legislador ordinario pueda introducir excepciones a la regla fundamental enunciada, excepciones que, como tales, son siempre de estricta interpretación, y no pueden por ello ser extendidas más allá de los casos que expresamente se prevén, posibilidad a la que responde el mencionado DPR 616/1977. La línea de separación entre la esfera de las relaciones internacionales en sentido estricto y aquella de las actividades que pueden desarrollar las Regiones viene, en consecuencia, trazada no tanto sobre la base de una diferencia cualitativa entre ellas, al estar ambas dirigidas a entablar relaciones con sujetos extranjeros al objeto de un mejor desarrollo económico, social y cultural de la comunidad nacional o regional, sino más bien sobre el tipo de resultados que pueden producir ${ }^{4}$.

Además, en su pronunciamiento, la Corte aporta la novedad de solucionar la conflictividad competencial que en ocasiones se venía planteando, al precisar que el límite de las relaciones internacionales de las Regiones no es un límite relativo a su incompetencia para ello, sino que, aparte de las atribuciones estatales sobre las relaciones internacionales en sentido estricto, debe entenderse como un límite al ejercicio de las competencias de las mismas ${ }^{5}$. En todo momento, la jurisprudencia constitucional es coincidente al reconocer que el sistema de las relaciones entre Estado y Regiones debe encontrar su base directa en el contexto constitucional y, en consecuencia, cada poder del Estado dirigido a incidir sobre tal autonomía va necesariamente unido a los principios constitucionales. Precisamente por ello, el sistema italiano se caracteriza por la búsqueda constante de un equilibrio entre la responsabilidad internacional del Estado y la salvaguarda de las autonomías garantizadas en la Constitución ${ }^{6}$, en el marco de una tendencia evolutiva en curso que puede desembocar en una reforma constitucional.

\section{LA ACCIÓN EXTERIOR DE LAS REGIONES Y PROVINCIAS AUTÓNOMAS}

Como se ha señalado, la Corte Costituzionale, en el tratamiento del alcance de la actividad regional en el extranjero, coloca en el centro de la discusión el principio de la unidad e indivisibilidad de la República italia-

\footnotetext{
${ }^{4}$ CARETTI, «Le attività di mero rilievo internazionale delle Regioni tra previa intesa e previo asenso governativo», en Giurisprudenza Costituzionale, 1992, págs. 4312-4313.

${ }^{5}$ CARETTI, ob. cit., pág. 4313.

${ }^{6}$ AgostinI, «Líneas de evolución de la acción de las Regiones italianas en el exterior y de su participación en el proceso de adopción de decisiones comunitarias», en La acción exterior y comunitaria de los Länder, Regiones, Cantones y Comunidades Autónomas, Vitoria, 1994, pág. 22.
} 
na, relacionándolo a partir de su Sentencia 46/1961, de 11 de julio, con la exclusiva subjetividad internacional del Estado, que no admite fragmentación o pluralidad alguna en la titularidad de la política exterior, lo que posteriormente ha seguido declarando en otros pronunciamientos (entre otras, en sus Sentencias 187/1985, de 28 de junio; 179/1987, 564/1988, de 19 de mayo; 42/1989, de 14 de febrero; 256/1989, de 18 de mayo, y 425/1995, de 12 de septiembre).

No obstante, el carácter centralista de tales principios no excluye una cierta potestad de las Regiones, incluidas las de autonomía especial y las Provincias autónomas ${ }^{7}$, para desarrollar en el exterior determinadas actividades, que bien pueden ser de tipo «promocional», o bien de «mero alcance internacional», según distinguió posteriormente en su ya citada Sentencia 179/1987. Procede, por tanto, singularizar a continuación ambos tipos de actividades.

\section{Las actividades promocionales}

Las actividades calificadas como «promocionales» venían contemplándose en el artículo 4.2 del DPR 616/1977, en el que se dispone que las Regiones pueden desarrollar en el exterior actividades de tal naturaleza relativas a la materia de su competencia, previo consentimiento del Gobierno y en el ámbito de las directrices y de la coordinación por él establecidas. Estas actividades, en cuanto ligadas de modo directo con las materias propias, hacen referencia a cualquier comportamiento directo relacionado con las mismas dirigido al desarrollo económico, social y cultural en el territorio del ente regional. Además, en su ejercicio debe garantizarse, en todo caso, una indispensable coherencia con la política exterior perseguida por el Estado (Sentencias 179/1987, 739/1988, de 30 de junio, y 472/1992, de 24 de noviembre), a lo que ayuda el riguroso ámbito de la potestad regional.

Un supuesto de este tipo de actividades lo constituye el tratado por la Sentencia 187/1985, de 28 de junio, que se enfrentaba al caso de un Protocolo de colaboración entre la Región del Valle de Aosta y la Región somalí de Basso Scebelli ${ }^{8}$. En esta ocasión, la Corte procedió a su anula-

\footnotetext{
${ }^{7}$ Las Regiones con Estatuto Especial o de autonomía especial comprende a las dos islas, Sicilia y Cerdeña, y a las Regiones fronterizas del Valle de Aosta, Trentino-Alto Adigio y Friuli-Venecia, entes que señaladamente se han visto afectados más a menudo por las decisiones de la Corte Constitucional en esta materia.

${ }^{8}$ La Región del Valle de Aosta posee un Estatuto especial por el que se le confiere personalidad jurídica propia, delegándose la mayoría de las funciones estatales a la misma, de modo que el Estado tan sólo tiene en la Región como órganos locales propios los tributarios y los de policía.
} 
ción, por cuanto tal Protocolo constituía un verdadero acuerdo afectante a diversas materias en las que el Estado poseía atribuciones - la industria metalúrgica, el sector zootécnico y el turístico, la artesanía y la sanidad-, realizado sin subordinar su eficacia a la autoridad estatal. Es más, podía producir efectos en las relaciones internacionales entre la República Italiana y la República Democrática de Somalia, razón por la que invadía la competencia reservada al Estado. El acuerdo impugnado, estimó, se estipuló fuera de cualquier coordinación con la política exterior estatal, además de que no se trataba la pretendida de una actividad promocional, sino de una verdadera y propia asistencia a un país en vías de desarrollo.

\section{Las actividades de mero alcance internacional}

La Corte distinguirá en su Sentencia 179/1987 tales actos de las consideradas «actividades de mero alcance internacional», que no son, en ningún modo, reconducibles al ámbito de las relaciones internacionales en sentido estricto. La decisión de la Corte viene a refrendar una praxis ya común, valorada favorablemente también en las decisiones de la jurisdicción administrativa, consistente en la frecuencia con que se pueden encontrar en el ámbito de la realidad internacional algunas actividades de diverso contenido conjuntamente realizadas por las Regiones y por otros entes extranjeros, normalmente homólogos a ellas, que tienen por objeto finalidades distintas a las promocionales.

De más incierta clasificación, estas actividades pueden poseer varios contenidos, y así es posible incluir las que tengan por objeto finalidades de estudio o de intercambio de información en materia técnica, o bien la previsión de actuaciones dirigidas a agilizar el progreso cultural y económico en el ámbito regional, e incluso la enunciación de propósitos tendentes a armonizar unilateralmente las respectivas actuaciones (pueden consistir, por ejemplo, en viajes de estudio, participación en convenios, visitas comerciales, etc.). Si bien la variedad de la materia no permite realizar una precisa clasificación, se puede destacar que, en todo caso, se trata de actividades que no afectan al territorio nacional en su totalidad, perteneciendo por ello a la iniciativa de los entes locales, y que son insusceptibles de incidir sobre la política exterior perseguida por el Estado o de determinar responsabilidad de cualquier género a cargo del mismo. Por el contrario, se limitan a prever el intercambio de información útil o bien la profundización de conocimientos en materias de interés común, o a enunciar aspiraciones compartidas, proponiéndose favorecer unilateralmente su realización mediante actos propios o, como mucho, mediante solicitu- 
des cursadas a los competentes órganos nacionales. Por ello mismo, entiende la Corte que no existe obstáculo alguno en el sistema constitucional italiano para reconocer la legitimidad de tales actividades, debiendo ser excluidas si presentan el riesgo de perjudicar a los intereses del Estado (Sentencia 564/1988).

La Corte ha ido delimitando en diversas ocasiones las actividades que entran o quedan fuera de las referidas relaciones internacionales de competencia estatal y de las actividades de mero alcance internacional de las Regiones. Así, ha considerado en su Sentencia 251/1993, de 27 de mayo, que un proyecto de Ley de la Región de Liguria sobre normas en materia de emigración - específicamente, la previsión de la asignación de contribuciones regionales dirigidas a sostener económicamente las asociaciones y organizaciones más representativas constituidas en el extranjero para el desarrollo de actividades a favor de los emigrantes ligures- no interfería en la esfera de las relaciones internacionales reservadas al Estado. Ello en razón de que, por una parte, no puede ser negada la presencia de un interés regional en este hecho y, por otra, que los organismos a los que se dirige la ayuda no son sujetos de Dereçho Internacional ni resultan dotados de poderes susceptibles de originar la responsabilidad internacional del Estado. Por su parte, la Sentencia 42/1989, de 14 de febrero, se refiere a una Declaración conjunta de la Región de Lombardía con el Land Baden-Wurttemberg, sobre materias que pudieran plantear un conflicto competencial -investigación científica, transferencia de tecnología, promoción del diseño, cooperación económica transfronteriza, etc. - . En esta ocasión, se considera legítima tal Declaración, por cuanto no trata de materias en sentido estricto, sino de metodologías de adquisición de conocimientos técnicos.

Por el contrario, en el caso de la Sentencia 290/1993, de 24 de junio, que juzgaba una declaración de intereses suscrita entre la Región de Cerdeña y la República Checa, consideró que el ente con el cual se relacionaba no era homólogo a la Región y que el contenido del acto - en gran parte sobre cooperación comercial- no estaba comprendido entre las materias reservadas a las competencias regionales al no ser ni un acto promocional ni de mero alcance internacional. Igualmente, en relación con la negociación y suscripción de una declaración de intereses entre la Región de Sicilia y la República de Túnez, consideró en su Sentencia $212 / 1994$, de 2 de junio, que la pretensión sobrepasaba la esfera de competencia regional, al tratarse de un acuerdo bilateral en materia de programación turística, que preveía la apertura en los respectivos territorios de representaciones de las partes encargadas de promover turismo y favore- 
cer iniciativas tendentes a incrementar los flujos turísticos provenientes de terceros países, con posibles implicaciones en el sector de la inmigración clandestina. En este caso, las partes tampoco eran homólogas, lo que superaba los límites propios de los actos de mero alcance internacional.

Otro supuesto es el tratado por la Sentencia 53/1996, de 27 de febrero, sobre una Ley de la Región del Valle de Aosta que disciplinaba las intervenciones de cooperación y solidaridad con los países en vía de desarrollo, regulando las formas y modalidades para hacerlo en el sentido de la Ley estatal 49/1987, de 26 de febrero, sobre cooperación de Italia con tales países, que califica dicha acción como parte integrante de la política exterior del Estado y establece el ámbito de intervención de las Regiones, de las Provincias autónomas y otros entes locales, ámbito que resultaba sobrepasado por algunos preceptos de la Ley regional, que por ello son anulados. También decidió en este sentido en el caso de la Sentencia 256/1989, de 18 de mayo, sobre un referéndum convocado por la Región de Cerdeña sobre, entre otras cuestiones, la presencia de bases militares extranjeras en su territorio. Dada la doctrina de la Corte de que el carácter unitario e indivisible de la República condiciona y subordina la autonomía regional, en la cual no puede ser incluida la potestad de decidir la instauración y la gestión de las relaciones internacionales, y el hecho de que la defensa militar es prerrogativa de los órganos estatales (artículos 11 y 52 de la Constitución italiana), decide que no es competencia de la Región convocar tal referéndum.

En cualquier caso, la diferenciación entre ambos tipos de actividades, que es una atribución del legislador estatal y de la Corte Constitucional, no carece en principio de relevancia, por cuanto según la calificación que se le otorgue a una determinada iniciativa, necesitará para llevarse a cabo del cumplimiento de unos específicos requisitos, que se concretan en la información previa al Gobierno de la República y el consentimiento, en distintos grados, que éste debe prestar para que la actividad regional pueda realizarse, como a continuación exponemos.

\section{EL REQUISITO NECESARIO DE LA COMUNICACIÓN PREVIA AL GOBIERNO DE LA REPÚBLICA}

En cada supuesto, se trate se realizar actividades promocionales o de mero alcance internacional, el Gobierno debe ser puesto al corriente, con razonable anticipación, de la actividad que tiene previsto desarrollar la Región en el exterior, exponiendo adecuadamente el contenido del acto 
que se proponga cumplir. La información suministrada por las Regiones se hace necesaria para que sea posible llegar a un acuerdo (intesa) entre aquella y el Gobierno, en el caso de las actividades promocionales, o bien para que éste otorgue su consentimiento (assenso), en el caso de las de mero alcance internacional.

En ambos casos, la información y la conformidad del Gobierno deben tener carácter previo a la ejecución de la actividad que se pretenda llevar a cabo, para que el mismo pueda realizar una valoración adecuada de la iniciativa y controlar su conformidad con las directrices de la política internacional, y quede así excluido el peligro de un perjuicio a los intereses nacionales. Tras esta evaluación, puede imponer su prohibición o, en su caso, la modificación de alguno de sus términos (en este sentido, entre otras, Sentencias 179/1987, 472/1992, 124/1993, 204/1993, de 29 de abril, y 425/1995).

Hay que aludir al respecto a la necesaria exigencia de unidad en las decisiones sobre las finalidades a perseguir por el Estado, exigencia que une la actividad política con la función del Gobierno, al que, en un sistema político como el italiano, compete indicar la orientación política y administrativa de todos los órganos constitucionales dotados de poder político (artículo 95 de la Constitución italiana), incluyéndose en aquélla la política general del Estado en las relaciones internacionales ${ }^{9}$. Así, en garantía de la unidad de la República, la Constitución y las leyes establecen instrumentos de diversa naturaleza dirigidos a adecuar los intereses regionales con los estatales, siempre con respeto a la autonomía regional, entre los que cabe señalar los poderes de dirección y coordinación que se atribuyen al Gobierno, dirigidos a orientar la actividad regional hacia fines compatibles con los objetivos constitucionalmente determinados ${ }^{10}$. No obstante, los límites del ejercicio de esta potestad estatal no están ni mucho menos totalmente claros, por lo que su alcance y justificación han provocado un intenso debate doctrinal y numerosos pronunciamientos de la Corte Costituzionale ${ }^{11}$. Últimamente, la Sentencia 18/1997, de 10 de febrero,

\footnotetext{
${ }^{9}$ En este sentido Coucolo, Principi di Diritto costituzionale, Milano, 1996, págs. 212 y ss., y Pızzorusso, Lecciones de Derecho Constitucional, I, Madrid, 1984, págs. 282-283.

10 Martines y Ruggers, Lineamenti di diritto regionale, Milano, 1997, págs. 143-144.

11 Ello porque la potestad de dirección y coordinación no se encuentra prevista constitucionalmente, por lo que el Gobierno introdujo en la Ley 281/1970, de 16 de mayo, de financiación para las Regiones, una reserva de tal función referida a las obligaciones internacionales, los objetivos de la programación económica y a exigencias de carácter unitario. Esta norma fue luego en parte sustituida por la Ley de delegación $382 / 1975$, que especificaba que tal potestad podía ser ejercitada sólo por ley o por deliberación del Consejo de Ministros o del Comité Interministerial para la programación económica por delegación de éste, y afectada en su contenido por la Ley delegada 616/1977,
} 
ha declarado que el poder estatal de dirección y coordinación se configura no ya como un límite ulterior a la autonomía de las Regiones, sino como expresión y manifestación de los limites constitucionalmente fijados. Así, dada la naturaleza constitucional de la autonomía regional, sólo a la Constitución y a la ley, en su desarrollo, compete la tarea de fijar las concretas dimensiones de la misma, su contenido y sus confines, siempre de acuerdo con las previsiones constitucionales.

La obligatoriedad mencionada se extiende a todos los entes locales, como fijó la Corte en un supuesto contemplado por la Sentencia 564/1988, de 19 de mayo, en el que las Provincias Autónomas de Trento y Bolzano ${ }^{12}$ recurrieron el Decreto del Presidente del Consejo de Ministros de 11 de marzo de 1980, dictado de acuerdo con èl DPR $616 / 1977$, sobre disposiciones de dirección y de coordinación para las actividades promocionales en el exterior de las Regiones en materia de su competencia, por entender que no debía serles de aplicación por tratarse de Provincias de autonomía especial. La Corte estableció que las funciones de dirección y coordinación constituyen actuaciones y desarrollo de un núcleo de fundamentales principios del ordenamiento constitucional, que deben vincular indistintamente a todos los entes autonómicos. Si bien se debe excluir que las funciones en discusión sirvan para introducir nuevos límites, además de los ya establecidos en el sistema constitucional, a la esfera de la autonomía regional, queda igualmente asegurado que las potestades de dirección y coordinación del Estado abarcan todo el ámbito de los poderes constitucionalmente garantizados a las Regiones y Provincias. Tales funciones, en cuanto justificadas por la necesidad de satisfacer las finalidades unitarias del ordenamiento, deben poder operar sin que quepa distinguir entre estatuto especial y estatuto ordinario, o entre tipos y grados de competencia de los entes autonómicos.

Esta obligación se hace derivar igualmente por la Corte Costituzionale del "principio de leal colaboración»" ${ }^{13}$, dirigido a superar las fisuras que en el ejercicio de las competencias del Estado y las Regiones pudieran

que delimita las funciones del Estado y precisa los puntos de mayor conflictividad con las Regiones. Asimismo, la Ley 400/1988 dispone que deben ser sometidos a la deliberación del Consejo de Ministros los actos de dirección y coordinación de la actividad administrativa de las Regiones. Véase sobre el particular Paladin, Diritto regionale, Padova, 1997, págs. 205 y ss., y MarTines y RUGGERI, ob. cit., págs. 346 y ss.

12 Las Provincias Autónomas de Trento y Bolzano, que cuentan con importantes atribuciones competenciales, componen la Región de Trentino-Alto Adigio.

13 Sobre el mismo, véanse por todas las Sentencias 135/1992, de 27 de marzo, y nota a la misma, y 188/1992, de 22 de abril. 
producirse, de modo que sus respectivas atribuciones se desarrollen de la manera más eficiente y coordinada posible ${ }^{14}$. Este principio también obliga al Estado a motivar la decisión que, en su caso, rechace el acuerdo o niegue su consentimiento previo, y a permitir a la Región elegir entre la renuncia a la iniciativa, la modificación de la misma en sentido conforme con las indicaciones estatales o el ejercicio de acciones de defensa de la esfera de sus propias competencias (como ha establecido en Sentencia 204/1993, de 29 de abril). De acuerdo con esta prerrogativa, en ocasiones se ha condicionado el acuerdo regional al cumplimiento de diversas indicaciones, como la reducción de la actividad en términos reales y financieros en la programación promocional anual de varios sectores en el extranjero de la Provincia de Trento, condicionamiento que la Corte declaró nulo en su Sentencia 26/1994, de 10 de febrero. La decisión de la Corte se basa en el carácter del acuerdo previo, que se justifica sólo por el control sobre la posible interferencia de la actividad regional o provincial en el extranjero con las directrices de la política exterior del Estado, por lo que aquella razón carece de fundamento legal sustancial, no pudiendo considerarse un acto de dirección ni de coordinación sobre la actividad promocional.

En particular, la jurisprudencia constitucional ha afirmado en diversas ocasiones que la suscripción de acuerdos con órganos o entes extranjeros sin que la Región haya informado previamente al Gobierno, y aun si lo ha hecho sin la necesaria profundidad, es un acto lesivo de la esfera de atribuciones estatales (Sentencias 204 y 425/1995), por lo que la omisión de una completa información por parte de la Región al Gobierno sobre el contenido de su iniciativa constituye razón suficiente para proceder a la anulación del acto, pues se sustrae a aquél de la oportunidad de evaluar debidamente el carácter y los fines de la iniciativa regional en el exterior.

En consecuencia, la Corte se ha visto obligada a anular actos regionales por faltar el necesario requisito de la previa información y acuerdo o consentimiento estatal. Así lo hizo en los casos mencionados anteriormente y resueltos por las Sentencias 290/1993 y 212/1994. Recientemente, en la Sentencia 343/1996, de 18 de octubre, se trató un supuesto en el que la Provincia Autónoma de Bolzano suscribió un acuerdo de colaboración con el Voivodato de Suwalki (Polonia), sin proporcionar comunicación alguna al Gobierno. Sin entrar en el examen de las materias incluidas en dicho tratado, ni en la forma en que el mismo se redactó, la Corte procedió a su anulación por entender que la falta de la previa información al

14 Martines y Ruggeri, ob. cit., pág. 126. 
Gobierno lesionaba la esfera de atribuciones del Estado. También es éste el caso de la Sentencia 739/1988, de 30 de junio, sobre un Protocolo de Amistad estipulado por la Región del Valle de Aosta con la Región francesa de Franca Contea, en el que tampoco se ofreció información previa al Gobierno sobre el mismo para que otorgara su consentimiento.

Debe señalarse que es el propio carácter de las actividades previstas el que determina la distinta naturaleza de este doble orden de requisitos, más formal en un caso que en el otro. Así, el previo acuerdo, por el hecho de referirse a actividad susceptible de incidir sobre las directrices de la política exterior del Estado, comporta un control más penetrante por parte del Gobierno, control que está destinado a realizarse mediante un consenso que necesariamente debe ser manifestado de forma explícita, y que resulta condicionante para que puedan iniciarse las pretensiones regionales a desarrollar en el exterior. Por el contrario, el previo consentimiento se caracteriza por su menor rigor formal, al no ser susceptibles de incidir las actividades para las que se solicita, por su propia naturaleza, sobre la política exterior del Estado o de determinar formas de responsabilidad a cargo del mismo. Por ello, este consentimiento puede ser manifestado incluso de forma tácita, por el transcurso de un tiempo prudencial una vez que la Región haya dado la oportuna información sobre las iniciativas programadas, aunque se ha señalado por la Corte la necesidad de que exista un término para la tramitación de las demandas de consentimiento, que podría ser fijado por el legislador o el Gobierno, eventualmente mediante un acto de dirección o de coordinación (Sentencia 472/1992).

La necesidad de esta información determina que la diferencia entre actividad promocional y actividad de mero alcance internacional pierda parte de su utilidad, lo que además se complica tanto por las contradictorias decisiones legislativas y de la jurisprudencia constitucional que aparecen a la hora de calificar una determinada actividad como afectante a la potestad internacional del Estado, como por el hecho de que los actos que se califican de irrelevantes necesiten del consentimiento del Gobierno, contradicciones interpretativas que ha señalado parte de la doctrina ${ }^{15}$.

Esta situación ha provocado posturas como la sustentada en ocasiones por el Gobierno italiano, cuando ha entendido que es necesario el previo acuerdo para realizar no sólo actividades promocionales en el extranjero relativas a la materia de su propia competencia, sino también para todas las iniciativas que pudiera comprender la actividad de mero alcance inter-

15 Así, De FIORES, «Riserva allo Stato dei rapporti internazionali e ruolo delle Regioni. Le nuove prospettive del «potere esterom», en Giurisprudenza Costituzionale, 1996, pág. 3013. 
nacional ${ }^{16}$. Ante esta postura reaccionaron diversas Regiones, por lo que la Corte Costituzionale se vio obligada a precisar en su Sentencia $472 / 1992$ el significado de la atribución del previo consentimiento gubernativo, además de clarificar si el mismo debe ser considerado un requisito análogo al del previo acuerdo o bien si se le debe atribuir una condición más flexible, donde toma claramente posición por la tesis del distinto significado de los dos requisitos, con base en la diferente actividad para los que se precisan ${ }^{17}$.

Hay que señalar, finalmente, que la evolución en la concepción de esta materia experimentada a partir de las declaraciones de la Corte Costituzionale, y el mayor protagonismo de los entes regionales en el seno y en las políticas de la Comunidad Europea, han hecho desaparecer la anterior asimilación de las relaciones comunitarias con las relaciones internacionales, lo que se ha llevado a cabo por medio del Decreto del Presidente de la República de 31 de marzo de 1994, que suprime en dicho ámbito los anteriores requisitos. Además, ha establecido explícitamente la diferenciación entre aquéllas en sentido estricto y las actividades exteriores de las regiones que, fuera de tales casos, son plenamente legítimas. Así, en su artículo 1 contiene una descripción de las actividades promocionales, distinguiéndolas de las actividades de mero alcance o relevancia internacional, de las que trata en su artículo 2, aunque no se refiere en ningún momento a los contenidos de tales actividades exteriores, sino que se ocupa sobre todo de fijar las normas procedimentales para llevar a cabo la valoración de dichas actividades por los órganos estatales, con el fin de otorgar el acuerdo o el consentimiento, e indica los criterios que inspiran dicha valoración.

Esta norma, adoptada como «Acto de dirección y coordinación en materia de actividad en el exterior de las regiones y provincias autónomas», se dirige a adecuar el contenido del mencionado DPCM de 11 de marzo de 1980, que dictaba disposiciones de dirección y coordinación de tales actividades, al desarrollo jurisprudencial del ordenamiento italiano y del comunitario, sustituyéndolo completamente. Respecto a las actividades comunitarias, permite a las Regiones que establezcan relaciones con or-

\footnotetext{
${ }^{16}$ Lo que realizó la Presidencia del Consejo de Ministros mediante Nota de 4 de enero de 1992, que, a los fines del reconocimiento de la necesidad del previo acuerdo del Gobierno con la Región, equiparó las actividades de mero alcance internacional a las actividades promocionales desarrolladas en el extranjero por las Regiones.

17 La Corte declara que no procede reclamar a la Región, en este caso Umbria, el previo acuerdo previsto en el artículo 4.2 DPR 616/1977, para una actividad de mero alcance internacional, anulando la Nota de la Presidencia del Consejo de Ministros de 4 de enero de 1992.
} 
NOTAS SOBRE LA ACCIÓN EXTERIOR DE LAS REGIONES...

ganismos, oficinas e instituciones comunitarias, como se ha dicho sin el requisito previo de la comunicación al Gobierno. No obstante, siguiendo en parte la línea hasta ese momento mantenida, se constriñe el ámbito de tales relaciones al de las cuestiones que directamente les afecten y a las actividades preparatorias de información y documentación, en ejecución de la política comunitaria determinada por el Estado. 


\section{Reseñas de legislación y jurisprudencia}


